\section{Tendência da mortalidade por câncer do útero no Município de São Paulo entre 1980 e 1999}

\author{
Mortality trends from uterine cervical cancer \\ in the city of São Paulo from 1980 to 1999
}

\section{Departamento de Medicina Preventiva, Faculdade de Medicina, Universidade de São Paulo, São Paulo, Brasil. \\ Correspondência Luiz Augusto Marcondes Fonseca Departamento de Medicina Preventiva, Faculdade de Medicina, Universidade de São Paulo. Av. Dr. Arnaldo 455, São Paulo, SP 01246-903, Brasil. luizaugusto.fonseca@ hcnet.usp.br}

\begin{abstract}
Uterine cervical cancer shows a higher incidence in some Brazilian cities. It is a common cause of death in women from developing countries, despite the longstanding availability of an effective screening test, the Pap smear. This study aimed to evaluate the temporal trends of crude, age-adjusted, and age-specific mortality rates from cervical cancer, endometrial cancer, and cancer of the uterus not otherwise specified (NOS) in the city of São Paulo from 1980 to 1999. Results showed a slight reduction in cervical cancer rates, a decrease in NOS uterine cancer rates, and an increase in endometrial cancer mortality rates. The fall in mortality from NOS uterine cancer indicates an improvement in diagnostic accuracy and quality of information on death certificates and may point to an increase in coverage of cervical cancer screening using the Pap smear.
\end{abstract}

Cervix Neoplasms; Cervical Smears; Mortality
Luiz Augusto Marcondes Fonseca 1 Adriana de Souza Ramacciotti 1 José Eluf Neto 1

\section{Introdução}

O câncer do colo do útero é uma das neoplasias malignas mais freqüentes, particularmente nos países em desenvolvimento. Estima-se que em 1990 ocorreram 371.200 casos novos de câncer invasivo de colo uterino em todo o mundo, representando quase $10 \%$ de todas as neoplasias malignas no sexo feminino 1 . Do total de casos de câncer cervical, $78 \%$ teriam ocorrido nos países em desenvolvimento.

No volume mais recente (VII) do livro Cancer Incidence in Five Continents, publicado pela Agência Internacional para Pesquisa em Câncer (IARC) 2, aparecem três registros populacionais de câncer do Brasil. As três cidades incluídas - Belém, Goiânia e Porto Alegre - estão entre os 20 registros com maiores incidências de câncer de colo uterino, sobretudo Belém, com 64,5 (2ㅇ), e Goiânia, com 37,1 (6o) (taxas ajustadas por idade por 100 mil mulheres). No Estado de São Paulo, estudo de incidência abrangendo 18 cidades do interior, enfocando o ano de 1991, constatou que o câncer do colo do útero era o segundo mais incidente em mulheres, excluindo-se o câncer de pele 3 .

No tocante à mortalidade, são estimados 230 mil óbitos anuais por câncer de colo uterino em todo o mundo, $80 \%$ dos quais em países em desenvolvimento 4. Entre estes, os maiores coeficientes são encontrados em países da África e da América Latina. 
No Estado de São Paulo, em 1993, o câncer do colo do útero ocupava a segunda posição como causa de morte feminina por câncer, suplantado apenas pelo câncer de mama 5

Comparado a outras neoplasias, o câncer cérvico-uterino apresenta uma característica importante: há longo tempo dispõe-se de um exame de rastreamento da doença, o teste de Papanicolaou, capaz de detectá-la em fase incipiente e curável com medidas relativamente simples. A grande redução da incidência e da mortalidade, observada desde o início dos anos 60 na maioria dos países desenvolvidos, é atribuída à prática de Papanicolaou 6 .

No Brasil, há poucos estudos sobre cobertura do teste de Papanicolaou em amostra aleatória da população. Em um estudo conduzido na cidade de São Paulo em 1987, 69\% das mulheres de 15 a 59 anos relataram pelo menos um teste na vida 7 .

Neste estudo, procurou-se analisar a evolução da mortalidade por câncer do colo do útero no Município de São Paulo em um período de 20 anos (1980-1999). Esta tendência ao longo do tempo permite avaliar de forma indireta a evolução do grau de cobertura do teste de rastreamento. Como a neoplasia de útero é muitas vezes registrada sem discriminar entre cérvix e corpo, foi também analisada a evolução da mortalidade por câncer do corpo do útero e por câncer do útero sem especificação de localização.

\section{Material e métodos}

Os dados brutos correspondentes aos óbitos de mulheres residentes no Município de São Paulo, cuja causa básica declarada fosse câncer do colo do útero, do corpo do útero, ou de partes não especificadas do útero, foram obtidos diretamente da Fundação Sistema Estadual de Análise de Dados Estatísticos (Fundação SEADE). O período enfocado na pesquisa foi de 1980 a 1999, no qual duas diferentes revisões da Classificação Internacional de Doenças (CID) estiveram em vigor: de 1980 a 1995, a 9ạ revisão (CID9), e de 1996 a 1999, a 10a revisão (CID-10). Na CID-9, os códigos correspondentes às categorias de interesse eram 180, para câncer do colo do útero, 182 para câncer do corpo do útero, e 179 para câncer do útero de outras localizações ou de localização não especificada, no útero. Os respectivos códigos da CID-10 são C53, C54 e C55 8,9.
Os dados relativos à população do Município de São Paulo foram obtidos junto à Fundação Instituto Brasileiro de Geografia e Estatística (IBGE), para os anos censitários (1980 e 1991) e para o ano de 1996 (contagem da população) 10,11,12; as estimativas intercensitárias foram calculadas pela Fundação SEADE, com base nas informações provenientes dos censos e dos estudos sobre migração e mortalidade.

Com as informações acima calcularam-se as taxas de mortalidade brutas totais, ajustadas por idade e idade-específicas, para as três localizações e para todos os anos em estudo. Para o cálculo das taxas ajustadas por idade utilizouse o método direto, tendo como população padrão a população "mundial" 13.

Para avaliar as tendências das taxas de mortalidade ao longo do tempo empregou-se a técnica de regressão linear, para cujo cálculo empregou-se o programa estatístico STATA, versão 5.0. Para a apresentação das figuras que expressam as tendências calcularam-se médias móveis de período 3 para cada localização de câncer e faixa etária. Foi realizada, também, análise gráfica da evolução da mortalidade segundo o ano de nascimento e a idade, tendo sido estudadas cinco coortes de mulheres nascidas nos decênios iniciados entre 1911 e 1951. Para cada decênio de nascimento e faixa etária, foi feita análise das taxas de mortalidade em três pontos no tempo: 1980, 1990 e 1999.

\section{Resultados}

A mortalidade por câncer de colo de útero, avaliada pelas taxas ajustadas por idade, mantevese estável entre 1980 e $1999(\beta=-0,001, p=0,9)$, enquanto as taxas relativas ao corpo do útero apresentaram comportamento ascendente $(\beta=$ $0,032, p=0,001)$. Já a mortalidade atribuída a câncer do útero sem especificação de localização registrou nítido movimento descendente $(\beta=-0,085, \mathrm{p}<0,001$ ) (Figura 1$)$.

Ao se analisar o comportamento da mortalidade por câncer do colo do útero por idade, observou-se estabilidade nas faixas etárias dos 30 aos $39(\beta=-0,004, p=0,8), \operatorname{dos} 40$ a $49(\beta=$ $0,013, \mathrm{p}=0,8)$ e dos 50 a 59 anos $(\beta=0,019, \mathrm{p}=$ $0,7)$, tendência para a ascensão nas idades de 70 anos e mais $(\beta=0,313, p=0,07)$, e queda significativa na faixa etária dos 60 a 69 anos $(\beta=$ $-0,26, p=0,004)$. As taxas relativas ao câncer do endométrio mostraram-se estáveis e com valores muito baixos dos 30 a 39 e 40 a 49 anos, 
Mortalidade por câncer do colo do útero, corpo do útero e útero sem outras especificações,

São Paulo, Brasil, 1980-1999 (médias móveis).

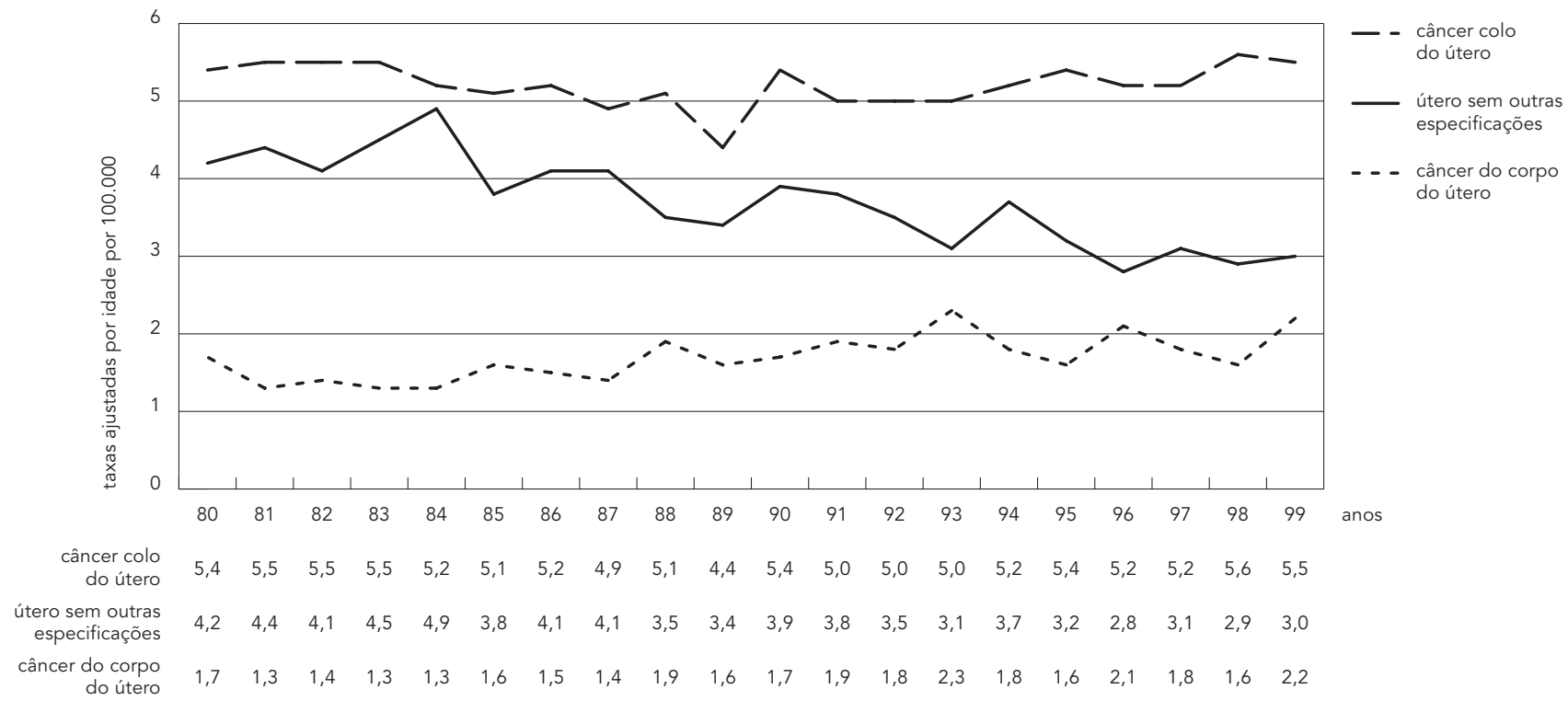

apresentaram tendência geral de aumento não significativo dos 50 até os 69 anos de idade, e aumento significativo nas idades de 70 anos e mais $(\beta=0,425, p=0,001)$.

A tendência temporal da mortalidade atribuída a câncer do útero não especificado foi decrescente em todas as idades menores que 70 anos, $\operatorname{com} \beta$ variando entre $-0,050$ e $-0,455$ e $\mathrm{p}=$ 0,01 ou menos. Na faixa etária dos 70 anos e mais, a tendência foi de estabilidade $(\beta=-0,019$, $p=0,9$ ). Cabe ressaltar que essa foi a única faixa etária na qual a mortalidade por câncer do útero não especificado suplantou aquela atribuída a câncer do colo do útero. A evolução da mortalidade por câncer do colo do útero, câncer do endométrio e câncer do útero não especificado, nas faixas etárias 30 a 39 anos, 40 a 49 anos, 50 a 59 anos e 60 a 69 anos é mostrada nas Figuras 2, 3, 4 e 5, respectivamente.

\section{Mortalidade segundo coortes de nascimento}

Procedeu-se a uma avaliação do comportamento das taxas de mortalidade segundo as coortes de nascimento, para câncer do colo e para cân- cer do útero não especificado, notando-se que os valores não seguem tendência definida, no caso do primeiro, e que caem, de modo consistente, em relação ao segundo (dados não apresentados).

\section{Discussão}

Neste estudo, a mortalidade atribuída especificamente a câncer do colo do útero, medida pelas taxas ajustadas por idade, não registrou alterações expressivas entre 1980 e 1999, na cidade de São Paulo; constatação que permanece válida quando se analisam as taxas específicas por idade, ou segundo as coortes de nascimento. Deve-se levar em conta que a mortalidade por câncer do colo do útero não é especificamente alta em São Paulo: conforme constatou este estudo, as taxas ajustadas por idade pela população mundial permaneceram sempre entre 5 e 5,5 por 100 mil mulheres, valores pouco superiores aos de vários países europeus, como Áustria, Dinamarca e Portugal, cujas taxas se situam entre 4 e 5 por 100 mil, menores que as da Argentina $(7,5)$, Chile $(10,6) \mathrm{e}$ do próprio Brasil $(11,5)$ e bastante inferiores às 
Mortalidade por câncer do colo do útero, corpo do útero e útero sem outras especificações,

faixa etária 30-39 anos, São Paulo, Brasil, 1980-1999 (médias móveis).

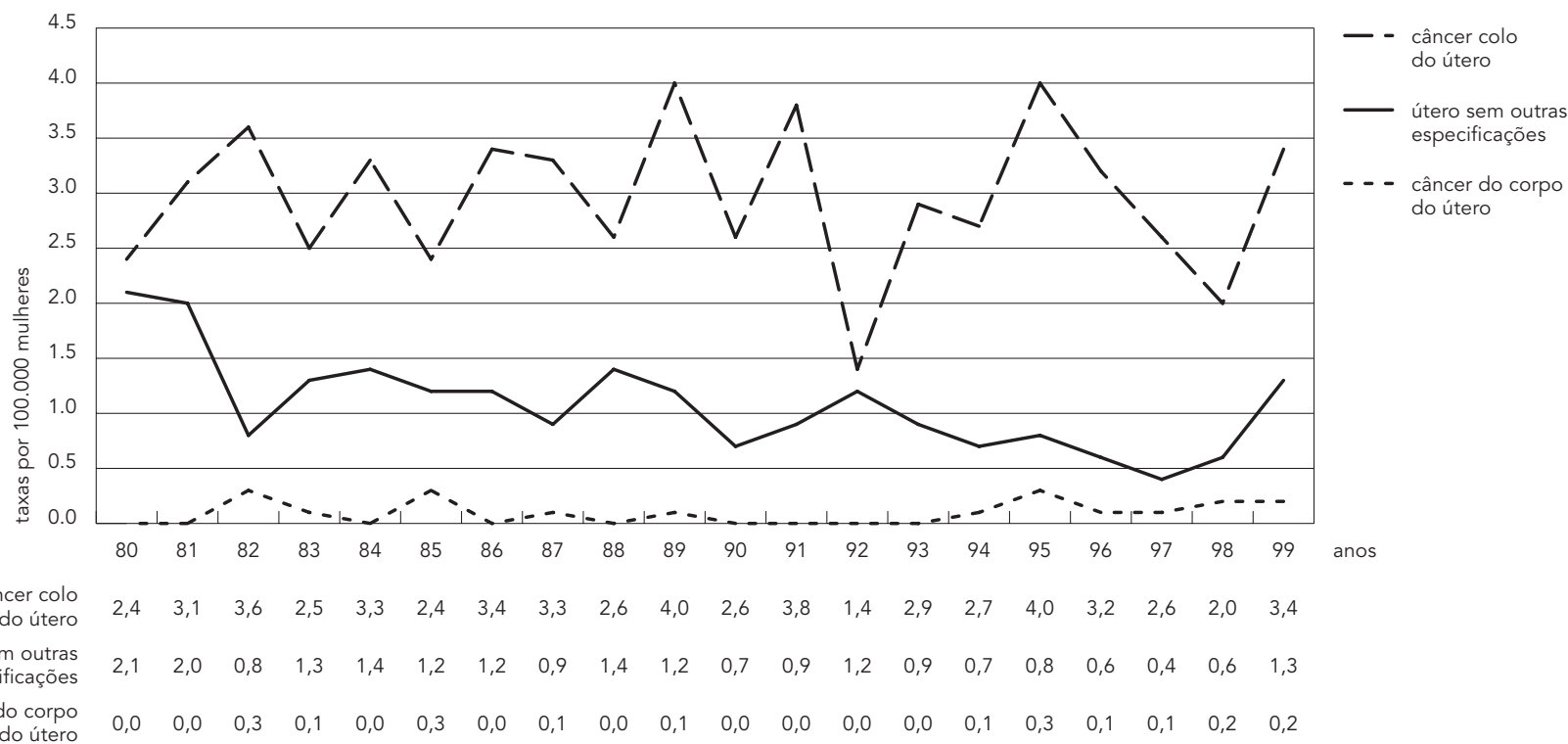

Figura 3

Mortalidade por câncer do colo do útero, corpo do útero e útero sem outras especificações,

faixa etária 40-49 anos, São Paulo, Brasil, 1980-1999 (médias móveis)

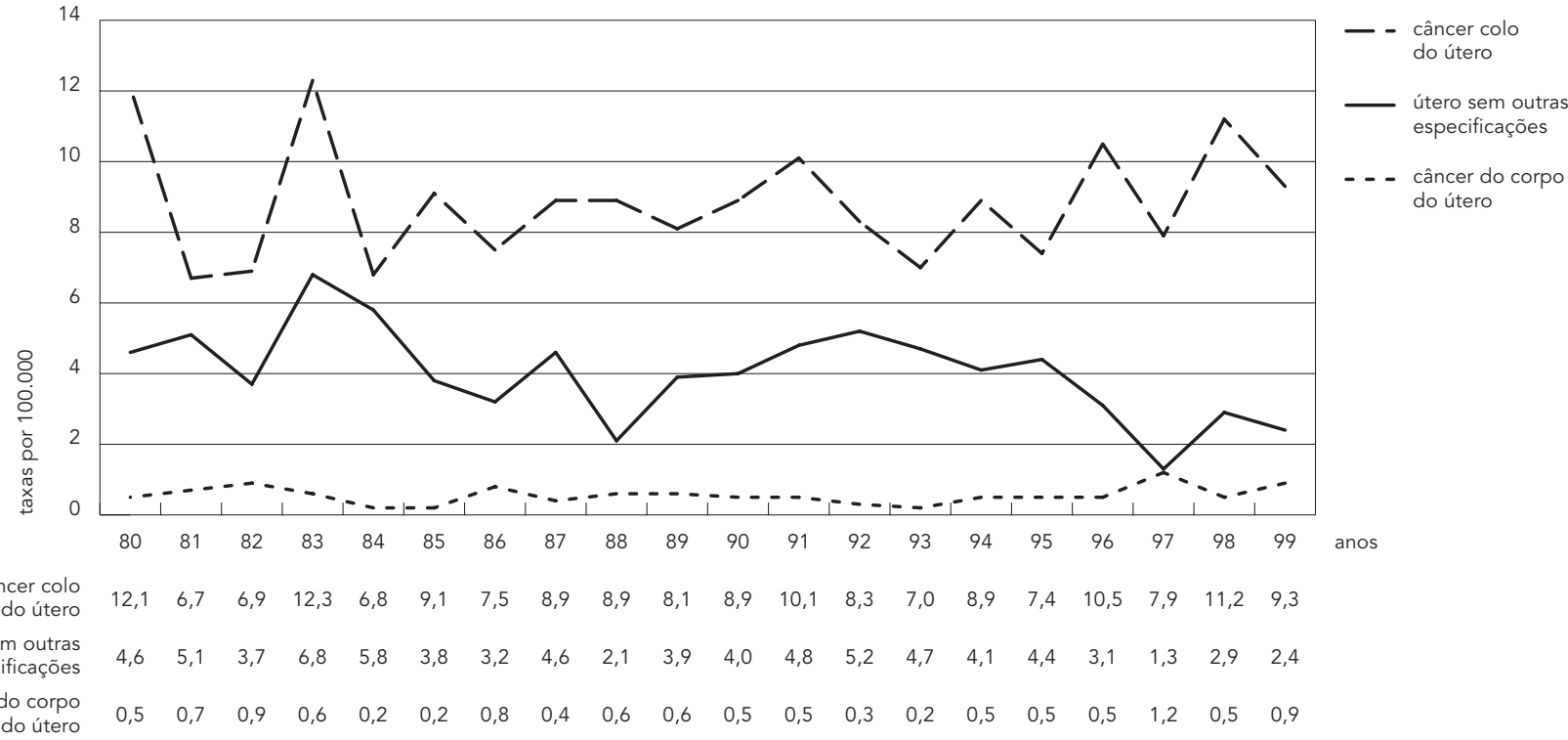


Figura 4

Mortalidade por câncer do colo do útero, corpo do útero e útero sem outras especificações, faixa etária 50-50 anos, São Paulo, Brasil, 1980-1999 (médias móveis).

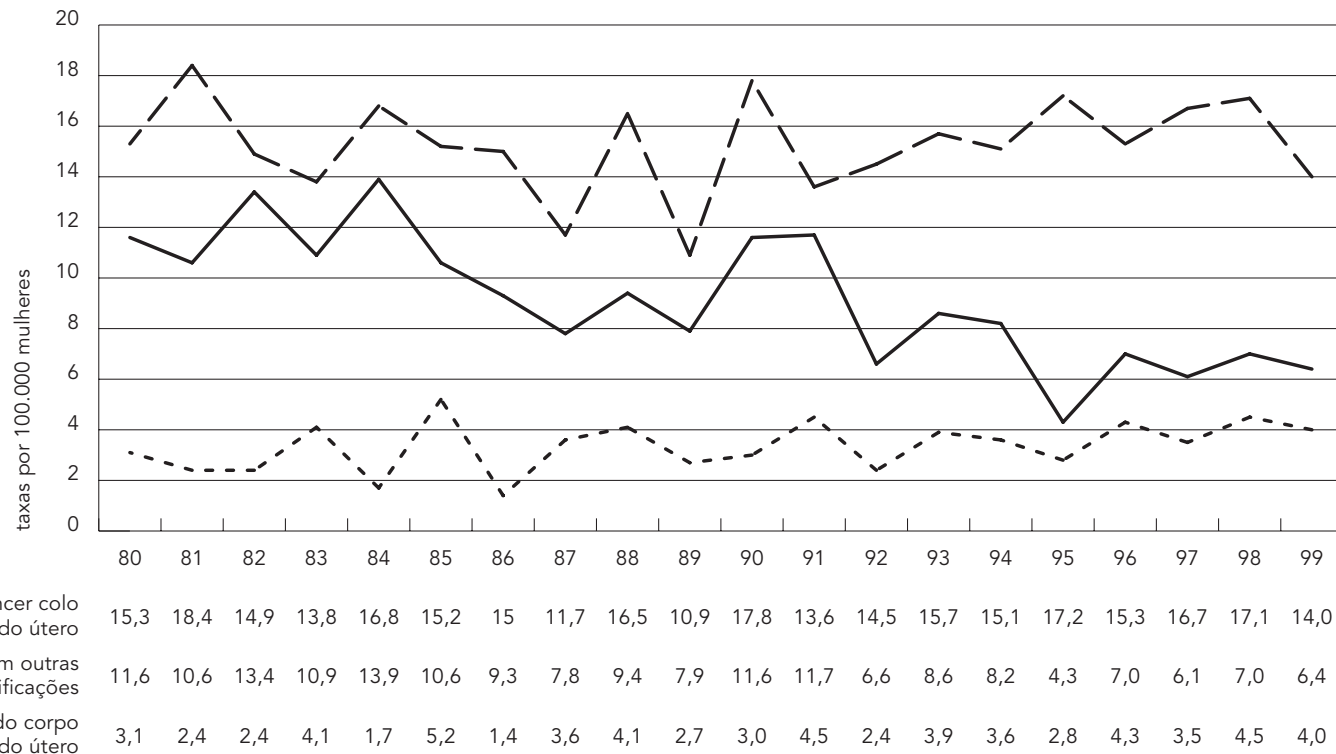

Figura 5

Mortalidade por câncer do colo do útero, corpo do útero e útero sem outras especificações,

faixa etária 60-69 anos, São Paulo, Brasil, 1980-1999 (médias móveis).

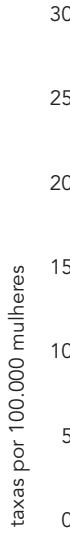

20
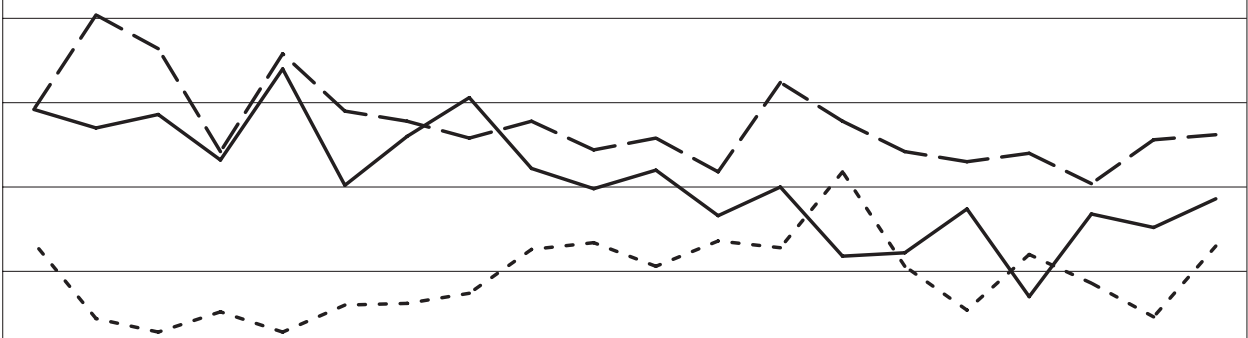

5

(1)

$\begin{array}{llllllllllllllllllll}80 & 81 & 82 & 83 & 84 & 85 & 86 & 87 & 88 & 89 & 90 & 91 & 92 & 93 & 94 & 95 & 96 & 97 & 98 & 99\end{array}$

câncer colo

do útero

útero sem outras

especificações

câncer do corpo

do útero $\begin{array}{llllllllllllllllllll}19,6 & 25,2 & 23,2 & 17,1 & 22,9 & 19,5 & 18,9 & 17,9 & 18,9 & 17,2 & 17,9 & 15,9 & 21,2 & 18,9 & 17,1 & 16,5 & 17,0 & 15,2 & 17,8 & 18,1\end{array}$

$\begin{array}{llllllllllllllllllll}19,6 & 18,5 & 19,3 & 16,6 & 22,0 & 15,1 & 18,0 & 20,3 & 16,1 & 14,9 & 16,0 & 13,3 & 15,0 & 10,9 & 11,1 & 13,7 & 8,5 & 13,4 & 12,6 & 14,3\end{array}$

$\begin{array}{llllllllllllllllllll}11,7 & 7,2 & 6,4 & 7,6 & 6,4 & 8,0 & 8,1 & 8,7 & 11,3 & 11,7 & 10,3 & 11,8 & 11,4 & 15,9 & 10,3 & 7,7 & 11,0 & 9,3 & 7,3 & 11,5\end{array}$
- - câncer colo

do útero

útero sem outras especificações

- - câncer do corpo do útero 
da maioria dos outros países sul-americanos e dos africanos, cujas taxas variam entre $13 \mathrm{e}$ mais de 20 por 100 mil mulheres 4 . Ainda assim, certamente há espaço para expressiva redução da mortalidade por esse câncer, visto que os países da América do Norte, o Japão, boa parte da Europa Ocidental e Israel, além de outros, apresentam valores que variam de 1,5 a 4,0 por 100 mil. Em boa parte desses países, a cobertura do teste de Papanicolaou é bastante alta, o que explica parte de sua baixa mortalidade 14.

Em relação ao câncer do corpo do útero, ou endométrio, verificou-se discreto, mas consistente aumento de taxas durante o período analisado. Vários estudos realizados em outros países têm apontado uma forte associação entre o uso de estrógenos e maior risco de câncer de endométrio 15,16, fato que também pode ter ocorrido entre as paulistanas, contribuindo para explicar o comportamento crescente da mortalidade por esse câncer.

No entanto, o achado que mais chamou a atenção foi a expressiva, contínua e persistente queda da mortalidade atribuída a câncer do útero sem especificação, a qual ocorreu durante todo o período analisado, em todas as idades, exceto nas acima dos 70 anos, e para todas as coortes de nascimento.

Em vista do quadro exposto acima, e procurando compatibilizar tais resultados com informações provenientes de estudo conduzido no Município de São Paulo em 1987, que apurou que $69 \%$ da população feminina entre 15 e 59 anos de idade já haviam feito pelo menos uma vez na vida o teste de Papanicolaou 7, dados corroborados em estudo recente 17 , levanta-se a hipótese de que boa parte da queda da mortalidade atribuída a câncer do útero sem especificação deve-se, realmente, à redução da mortalidade por câncer do colo do útero. Corrobora esta hipótese o fato de a queda das taxas iniciar-se já nas idades mais jovens, nas quais a mortalidade pela única outra localização uterina de câncer, a do endométrio, é praticamente nula. Outro achado que apóia aquela hipótese é a ausência de redução da mortalidade por câncer de útero não especificado na faixa etária mais alta, e o fato de este diagnóstico suplantar, nessa faixa, o de colo do útero, mais específico, o que está de acordo com a tendência geral de se registrar, na declaração de óbito das pessoas mais idosas, diagnósticos menos precisos 18. Este fato ressalta a importância de se incluir, nos estudos de mortalidade, uma análise do comportamento temporal da mortalidade pelas causas menos definidas, a exemplo de câncer de útero não especificado, abordado neste trabalho, ainda que estejam abrigadas em capítulos específicos da CID.

É provável que a expansão do acesso à atenção médica, incluído aí o próprio teste de $\mathrm{Pa}$ panicolaou, bem como à informação sobre a doença expliquem parte da queda da mortalidade entre as mulheres mais jovens, observada no período estudado; já a maior precisão na atribuição da causa básica de morte pode ser devida à maior disponibilidade de métodos diagnósticos em anos mais recentes.

\section{Resumo}

O câncer do colo do útero apresenta grande incidência em algumas cidades brasileiras e considerável mortalidade em países em desenvolvimento, não obstante a disponibilidade já antiga de teste de rastreamento. $O$ presente estudo visou avaliar a tendência da mortalidade por câncer de colo do útero, de corpo do útero e por câncer do útero não especificado, no Município de São Paulo, entre 1980 e 1999, por meio do exame das taxas brutas, idade-específica e ajustadas por idade. Os resultados mostraram discreta redução da mortalidade por câncer do colo do útero, queda da mortalida- de por câncer de útero não especificado e aumento da mortalidade por câncer do corpo do útero. Conclui-se que a queda da mortalidade por câncer do útero não especificado sinaliza uma melhora na precisão do diagnóstico clínico e na qualidade do preenchimento do atestado de óbito, e indica aumento de cobertura do teste de Papanicolaou.

Neoplasias do Colo Uterino; Esfregaço Cervical; Mortalidade 


\section{Colaboradores}

L. A. M. Fonseca contribuiu nas etapas de planejamento, análise e redação do manuscrito. A. S. Ramacciotti participou da coleta e processamento dos dados. J. Eluf Neto colaborou na concepção, análise e discussão do trabalho.

\section{Referências}

1. Parkin DM, Pisani P, Ferlay J. Estimates of the worldwide incidence of the twenty-five major cancers in 1990. Int J Cancer 1999; 80:827-41.

2. Parkin DM, Whelan SL, Ferlay J, Raymond L, Young J, editors. Cancer incidence in five continents. v. 7. Lyon: International Agency for Research on Cancer; 1997 (Scientific Publication 143).

3. Andreoni GI, Veneziano DB, Giannotti Filho O, Marigo C, Mirra AP, Fonseca LAM. Cancer incidence in eighteen cities of the state of São Paulo, Brazil. Rev Saúde Pública 2001; 35:362-7.

4. Ferlay J, Parkin DM, Pisani P, Globocan L. Cancer incidence and mortality worldwide in 1990. Cancer Bases no 3. Lyon: International Agency for Research on Cancer; 1998.

5. Fonseca LAM. Mortalidade por câncer no Estado de São Paulo: perfil atual, distribuição geográfica e tendências temporais [Tese de Doutorado]. São Paulo: Faculdade de Saúde Pública, Universidade de São Paulo; 1996.

6. Schiffman MH, Brinton LA, Devesa SS. Cervical cancer. In: Schottenfeld D, Fraumeni Jr JF, editors. Cancer Epidemiology and Prevention. 2nd ed. New York: Oxford University Press; 1996. p. 1090-116.

7. Organização Mundial da Saúde. Manual da Classificação Estatística Internacional de Doenças, Lesões e Causas de Óbitos - Nona Conferência de Revisão. São Paulo: Centro Brasileiro de Classificação de Doenças em Português; 1985.

8. Nascimento CM, Eluf Neto J, Rego RA. Pap test coverage in São Paulo Municipality and characteristics of the women tested. Bull Pan Am Health Organ 1996; 30:313-21.

9. Organização Mundial da Saúde. Classificação Estatística Internacional de Doenças e Problemas Relacionados à Saúde, 10ạ Revisão. v. 1. São Paulo: Centro Colaborador da OMS para a Classificação de Doenças em Português; 1995.
10. Fundação Instituto Brasileiro de Geografia e Estatística. Censo demográfico, São Paulo - 1980. v.1. Rio de Janeiro: Fundação Instituto Brasileiro de Geografia e Estatística; 1981.

11. Fundação Instituto Brasileiro de Geografia e Estatística. Censo demográfico, São Paulo - 1991. no 21. Rio de Janeiro: Fundação Instituto Brasileiro de Geografia e Estatística; 1994.

12. Fundação Instituto Brasileiro de Geografia e Estatística. Contagem da população 1996. Rio de Janeiro: Fundação Instituto Brasileiro de Geografia e Estatística; 1997.

13. Doll R, Cook P. Summarizing indices for comparison of cancer incidence data. Int J Cancer 1967; 2:269-79.

14. Quinn M, Babb P, Jones J, Allen E. Effect of screening on incidence of and mortality from cancer of cervix in England: evaluation based on routinely collected statistics. Br Med J 1999; 318:904-8.

15. Cushing KL, Weiss NS, Voigt LF, McKnight B, Beresford SA. Risk of endometrial cancer in relation to the use of low-dose, unopposed estrogens. Obstet Gynecol 1998; 91:35-9.

16. Grady D, Gebretsadik T, Kerlikowske K, ErnsterV, Petitti D. Hormone replacement therapy and endometrial cancer risk: a meta-analysis. Obstet Gynecol 1995; 85:304-13.

17. Pinho AA. Fatores associados à realização do teste de Papanicolaou entre mulheres em idade reprodutiva no Município de São Paulo [Dissertação de Mestrado]. São Paulo: Faculdade de Saúde Pública, Universidade de São Paulo; 2002.

18. Lu TH, Shau WY, Shih TP, Lee MC, Chou MC, Lin CK. Factors associated with errors in death certificate completion. J Clin Epidemiol 2001; 54:232-8.

Recebido em 20/Set/2002

Versão final reapresentada em 5/Mai/2003 Aprovado em 25/Set/2003 